\title{
Design of Nano-Manufacturing System over Traditional Manufacturing System
}

\author{
Sunil Kumar Jakhar, Deepanshu Arora, Sanjay Choudhary, Krishna Nandan, Amogh Sharma
}

\begin{abstract}
History has shown that in general either the science behind a manufacturing process leads to the development of new technology to enable it or a new technology is developed which then leads to scientific investigation into developing an understanding of the processes. This practice does not show any sign of wavering, the development of new Nano-manufacturing process has been demonstrated to be feasible in the laboratory, the focus should then ideally shift to turning it into a manufacturing process.
\end{abstract}

Index Terms-Nano- Manufacturing, High Level Metrics (HLMs), Low Level Metrics (LLMs).

\section{INTRODUCTION}

According to Lyons, "Nano-manufacturing can be defined as all manufacturing activities that collectively support an approach to design, control, modify, manipulate, and assemble nanometer scale objects and features for the purpose of fabricating a product or system that exploits properties seen at the Nanoscale." Currently, instruments for enabling Nano-manufacturing, now Nanoscale products must be able to be "produced" on a large scale to realize their full potential.

\section{NANOMANUFACTURING SYSTEM DESIGN}

A metric mapping process is introduced where in different types of manufacturing processes are compared based on set of high- and low-level metrics. Once nano processes is identified it must be compare to different macro-processes, and then the technology used by macro processes can be utilized as a road map for guiding and influencing the design of Figures nanomanufacturing machine. A method for isolating a definitive objective means of comparing nano and macro scale manufacturing processes are presented here to give design engineers a guide by which to create new manufacturing technology. This method used two taxonomic ranks are High Level Metrics (HLMs) and Low-Level Metrics (LLMs).

Sunil Kumar Jakhar, Assistant Professor, Department of Mechanical Engineering, Vivekananda Institute of Technology, Jaipur, India, 94143873933

Deepanshu Arora, Assistant Professor, Department of Mechanical Engineering, Vivekananda Institute of Technology, Jaipur, India, 7737283561 .

Sanjay Choudhary, Assistant Professor, Department of Mechanical Engineering, Vivekananda Institute of Technology, Jaipur, India, 9928774884.

Krishna Nandan, Assistant Professor, Department of Mechanical Engineering, Vivekananda Institute of Technology, Jaipur, India, 9828913593.

Amogh Sharma, Assistant Professor, Department of Mechanical Engineering, Vivekananda Institute of Technology, Jaipur, India, 7728041345 .

\section{A. High Level Metrics (HLMS)}

Different types of manufacturing processes can be defined as a model of manufacturing process by the following three characteristics.

- Type of process

- State of the workpiece material

- Nature of the processing energy

The type of process refers to two major diversions which are distinguished based on whether the workpiece is shaped, or its geometry modified during the process. Shaping processes change the workpiece shape or geometry, while non-shaping processes do not. Non-shaping processes can also refer to as treating process because they can be used to treat a material and modify its chemical or compositional characteristics. On the other hand, shaping processes can be divided into three distinct categories: mass-conserving, mass-reducing, and mass-joining. In micromanufacturing processes including milling, turning, and forging, example of non-shaping includes heat-treatment, quenching

The second characteristic, the state of the work piece material, refer to the physical state of the material while it is undergoing the specific shaping or non-shaping process. Whether the work-piece material is in a solid, liquid, granular, or vapor state, each of the different types of processing energies can be used to modify the material in some manner. This gives rise to the third defining characteristics of manufacturing processes. It should be noted that model only deals with shaping processes when describing the state of the workpiece and the third defining characteristics.

The nature of the processing energy is the third characteristics, and it generally takes one of the following three forms: mechanical, thermal, chemical. While there are many different forms of energy, including electrical and irradiative, these three are the most prevalent among manufacturing processes. While those processes which use irradiative energy to modify the work-piece material generally involve the use of heat transfer through irradiation.

\section{B. LOW LEVEL METRICS (LLMS)}

The low-level metrics used to characterize a nano manufacturing process including the previously described variable most used generally describe manufacturing processes they are not as important as high-level metrics, but these are process variables which refer to similarities among the properties of the manufacturing process.

Rate: Rate as described before, can have several different units in conventional manufacturing can be taken as the cycle time of the slowest machine in the manufacturing system or

Table 1: Different type of manufacturing processes 


\begin{tabular}{|c|c|c|}
\hline \multicolumn{3}{|c|}{$\begin{array}{l}\text { Classification of different types of manufacturing } \\
\text { processes as described by Todd }\end{array}$} \\
\hline $\begin{array}{l}\text { Type of process } \\
\text { (family) }\end{array}$ & $\begin{array}{l}\text { State } \\
\text { material }\end{array}$ & $\begin{array}{l}\text { Nature of process } \\
\text { Energy }\end{array}$ \\
\hline \multicolumn{3}{|c|}{ Shaping Process } \\
\hline 1. Mass-reducing & Solid & Mechanical \\
\hline 2. Mass-reducing & Solid & Thermal \\
\hline 3. Mass-reducing & Solid & Chemical \\
\hline $\begin{array}{l}4 . \\
\text { Mass-Conserving }\end{array}$ & Solid-Granular & Mechanical \\
\hline 5. Consolidation & Liquid/Plastic & Mechanical \\
\hline 6. Joining & $\begin{array}{l}\text { Solid (except } \\
\text { adjacent } \\
\text { surface) }\end{array}$ & Mechanical \\
\hline \multicolumn{3}{|c|}{ Non-shaping Processes } \\
\hline 7.Hardening & Solid & Chemical/Thermal \\
\hline 8. Softening & Preparation & Mechanical \\
\hline $\begin{array}{l}\text { 9.Surface } \\
\text { Treatment }\end{array}$ & Solid & Chemical/Thermal \\
\hline $\begin{array}{l}\text { 10.Surface } \\
\text { treatment }\end{array}$ & Solid & Chemical/Thermal \\
\hline
\end{tabular}

the rate at which parts or finished components are produced from the system. In terms of metrics, it is probably best assumed that rate taken as the total time required for the smallest individual work-piece unit to pass through the system. The smallest individual work-piece unit could be single aluminum casting, a monolith type fixture to which several parts are affixed, or single gold substrate sample on which ten thousand of nano-features are created.

Cost: The next metric considered in low-level comparison of manufacturing metrics, can be taken in terms of the cost of an individual machine to enable the process, or the cost per work-piece unit (the same work-piece unit above). It is essential that the low-level metrics be normalized to the same function unit as their macro scale counterparts so that a more accurate comparison of the metrics between manufacturing scales can be made, especially when considering cost. Even 0 after the decimal point increases cost dramatically; at the nanoscale this mean the different between finding a closely related metric and not finding any good matches.

Quality: When comparing low-level metric for macro and nano scale manufacturing processes the comparison must be scaled relative to the process. It would be better to evaluate processes on a qualitative quantity scale rather than on their ability to achieve a desired tolerance. In macro machining, quality is often referred to in terms of dimensional accuracy as well as the dimensional constancy from part to part this relative measure are useful in identifying dimensional capability of different processes as the drastic difference in scale would tend to skew any solid comparison.

Flexibility: In terms of comparison between nano and macro manufacturing processes, the simplest definition of flexibility is the best to use. Flexibility measures the ability of process is its ability to change associated variable. It can be used to describe how many different products are able to be manufactured by the process as well as its ability to achieve different standards of quality.

\section{METRIC MAPPING}

The matric mapping (METMAP) process is carried out in cycle, as much like a deterministic design using FRDPRRC [Functional requirement-FR + Design parameter-DPs + Analysis-A + Reference- $\mathrm{R}+$ Countermeasures-C] is utilized to chip away at a large design problem by tacking small nuggets. METMAP using 5 steps isolated macro manufacturing process which is the most like a single nanomanufacturing process. The 5 steps of METMAP are as following

- Process identification

- Comparison of High-level Metric

- Process physics

- Comparison of low-level metrics

- Identified of "Best" Candidate process

The first step in metric mapping is identified the nano processes that will be enable by any resulting manufacturing technology, as well as a several candidate macro scale processes.

These macro manufacturing processes are chosen at an individual discretion, and process as whole may take multiple iteration with different candidate process groups to yield results. After processes have been identified, the next step in the process is used to identify general similarities between nano and macro manufacturing process.

The second step of the comparison of HLMs, comperes the three characteristics of single nanomanufacturing process with each candidate micromanufacturing process to identify similarities between high level metrics of each process pair. When group of between 4 and 10 macro-manufacturing processes are identified by their HLMs to be like the nanomanufacturing processes, the physics of each process are the physics that drives a process is essential to accurately compare it to other manufacturing processes. After an understanding of each process is achieved, the next and perhaps most critical step is reached; comparison of LLMs. This is where the final candidate processes are identified before physical machinery and enable technology is evaluated. Once a macro process is identified as being like the nano process on this level, a design engineer can begin to get an idea of the type of technology which could be used to enable the desire nanomanufacturing process.

Next to this, the comparison of HLMs, here the 3 characters of a single nano manufacturing process with each candidate at macro manufacturing process identify the similarities between HLM of each process. After the identification of 4 to 10 macro-manufacturing processes identification like the nanomanufacturing process, the physis of each process are then described and evaluated to ensure the easy mapping or conversion.

After an idea of each process, the next important step must follow is compression of LLMs. At this stage final candidate process are identified before any micro to nano machinery enabling. The main target here is to fully identify the all the macro processes in the traditional manufacturing like 
the nano manufacturing for the easy conversion. After that we can design or bring the appropriate machine to enable the desired nanomanufacturing process. The traditional to the modern nanomanufacturing system conversion is detailed explain in the following figure.

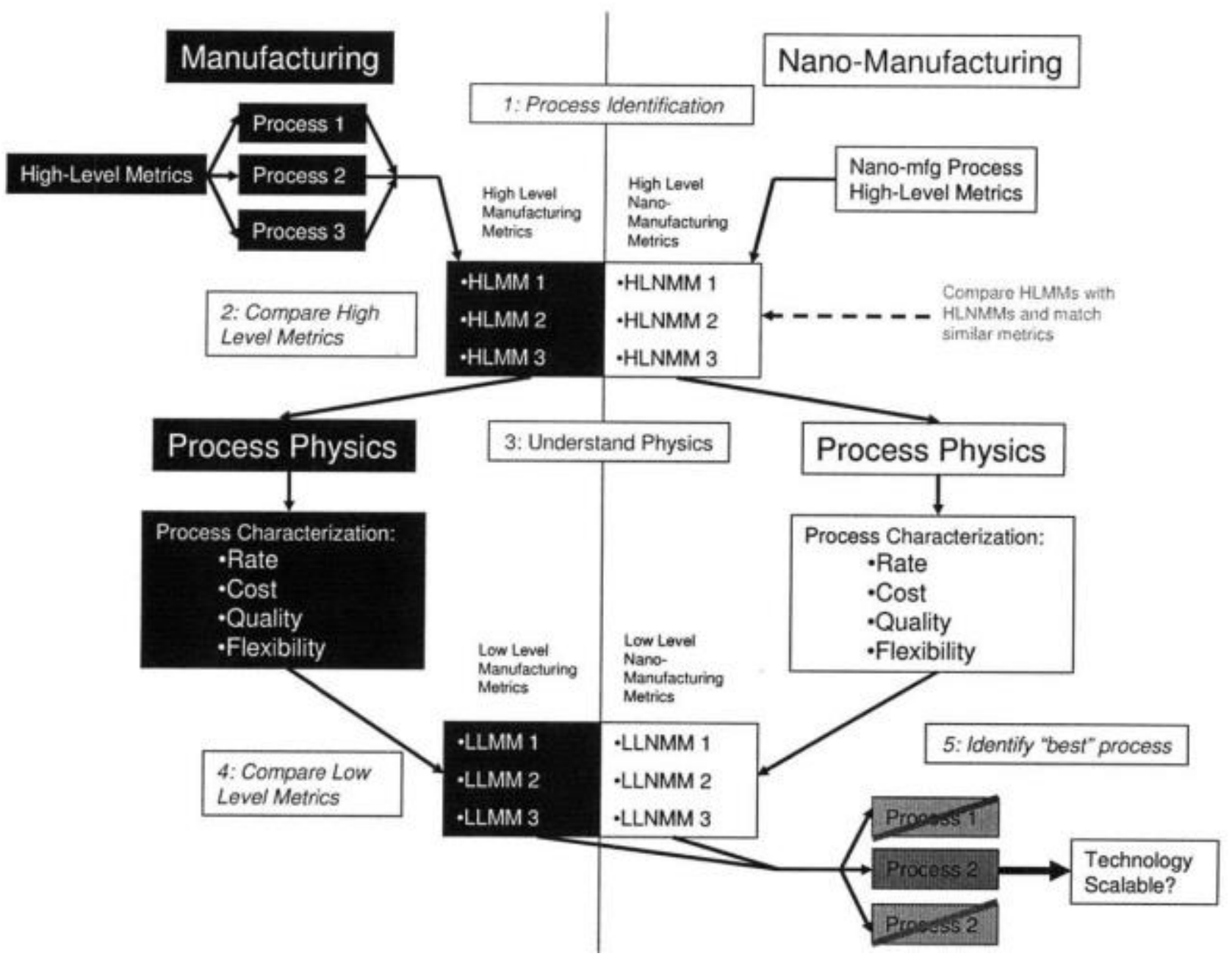

Figure 1: Matrix level Mapping of the traditional and the modern nanomanufacturing systems.

\section{CONCLUSION}

The present study provides a platform for the research and development industries, medicinal, chemical, other manufacturing industries to design the nano manufacturing facility for the innovations. Some time when the new product development is very important to manufacture or develop on its early stage of development, the traditional manufacturing does not support the design and development, due to sometime complexity and diversity of the product. This study explains the procedure to identification of the similar processes of traditional and nano manufacturing for the better identification of the process parameters and the required machines. After that, the level-based mapping support the design work for the modern nanomanufacturing by arranging the appropriate machines and the other required articles under the same. This will help in the investment for the design of new nano manufacturing facility, due to follow each and every constraint from scratch, this minimizes design cost as well as implementing cost of the organization.

\section{REFERENCES}

[1] A. Benilov, V. S. (2008). Micro and nano electrical discharge machining in microfluidics and micro nanotechnology. Institut des Nanotechnologies.

[2] Alexander H. Slocum, J. (2010). Design of Nanomanufacturing Systems. Massachusetts Institute of Technology.

[3] Budworth, D. D. (1996). Overview Of Activites on Nanotechnology and Related Technologies. Institute for prospective technological studies of the Europen Commission, Joint Ressearch Center.

[4] Gutowski, T. (July 8, 2002). Measuring Performance; Metrics for Time, Rate, Cost, Quality, Flexibility, and the Environment. Handout for MIT Course2.810; Manufacturing Processes and System.

[5] Wang, B. C. (2005). Road Map for the Controlled Synthesis of CdSe Nanowires, Nanobelts, and Nanosaws a Step Towards Nanomanufacturing. Advanced Materials.

Sunil Kumar Jakhar, currently working as assistant professor at Vivekananda Institute of Technology Jaipur. He done his M. Tech in Thermal and Fluid Engineering from IIT BHU in 2015, and B. Tech in Mechanical Engineering from GVSET Jaipur in 2010. He has total 09 years of experience in Teaching and Research.

Deepanshu Arora, currently working as assistant professor at Vivekananda Institute of Technology Jaipur. He done his M. Tech in Manufacturing System Engineering from MNIT Jaipur in 2012, and B. Tech in Mechanical Engineering from RGPV Bhopal in 2009. He has total 12 years of experience in Teaching, Research, and Industry. 
Sanjay Choudhary, currently working as assistant professor at Vivekananda Institute of Technology Jaipur. He done his M. Tech in Production Engineering from RTU Kota in 2021, and B. Tech in Mechanical Engineering from SKIT Jaipur in 2010. He has total 11 years of experience in Teaching and Research.

Krishna Nandan, currently working as Assistant professor at Vivekananda Institute of Technology Jaipur. He is pursuing M. Tech in Production Engineering from VGU Jaipur (2019-21) and B. Tech in Mechanical engineering from VIT-East Jaipur in 2016. He has total 5 years of experience in Teaching.

Amogh Sharma, currently working as assistant professor at Vivekananda Institute of Technology Jaipur. He is pursuing his M. Tech in Production Engineering from RTU Kota and completed his B. Tech in Mechanical Engineering from UPTU Lucknow in 2011. He has total 10 years of experience in Teaching, Research, and Industry. 